from Dr. M. P. Starr, University of California at Davis, and the other from Dr. Muriel Rhodes, University of Reading, Reading, England, who had received it from the British NCTC. The other culture, British NCTC no. 9433, was also sent by Dr. Rhodes. The origin of this culture is not known by us.

The results of typing using 20 bacteriophages are given in table 1 . The activity of bacteriophages for $P$. aeruginosa on these cultures is further evidence that $P$. polycolor cannot be distinguished from $P$. aeruginosa. The phage typing pattern shown by the three cultures of $P$. polycolor does not separate these organisms from approximately 250 strains of $P$. aeruginosa which have been tested. The results indicate that the typing pattern of PP2 has remained stable during maintenance in separate laboratories.

Since the strains of $P$. aeruginosa have shown a wide variety of phage typing patterns it seems unlikely that the nearly identical typing patterns demonstrated by these cultures will be found in all strains from plants but this will not be known until more organisms can be tested.

\title{
OCCURRENCE OF $\beta$-GALACTOSIDASE IN THE GENUS SHIGELLA
}

\author{
H. V. RICKENBERG \\ Department of Microbiology, University of Washington, Seattle, Washington
}

Received for publication May 2, 1960

Partial genetic homology between the genera Escherichia and Shigella has been demonstrated by phage mediated transduction of genetic characters between the two genera (Lennox, Virology, 1, 190, 1955; Adams and Luria, Proc. Natl. Acad. Sci. U. S., 44, 590, 1958) as well as by conjugational recombination (Luria and Burrous, J. Bacteriol., 74, 461, 1957).

One of the genetic markers employed in these experiments was the lac character. The genus Escherichia is characterized by its ability to ferment lactose; in the genus Shigella lactose fermentation does not occur in a majority of species and only after a prolonged delay in other species. In the transduction and recombination experiments quoted, shigella strains were employed which were altogether devoid of the ability to ferment lactose and in which the presence of a "cryptic" $\beta$-galactosidase (Rickenberg and Yanofsky, Bacteriol. Proc., 1954, 52) appeared to be excluded by the inability of bacterial extracts to hydrolyze the chromogenic galactoside $\boldsymbol{o}$-nitrophenyl- $\beta$-D-galactopyranoside (ONPG).

Recent work by the author suggests that a number of shigella strains, including Shigella dysenteriae strain Sh, which has been used in transduction and recombination experiments, do possess $\beta$-galactosidase activity, although they are incapable of lactose fermentation.

Cultures were grown on a medium consisting of one part nutrient broth and one part mineral medium to which glycerol had been added to a final concentration of 0.2 per cent. $\beta$-Galactosidase inducers were added as indicated in table 1. $\beta$-Galactosidase activity and protein concentration were determined as reported previously (Rickenberg, Yanofsky, and Bonner, J. Bacteriol., 66, 683, 1953).

Table 1 shows that considerable $\beta$-galactosidase activity is found in Shigella paradysenteriae strain Y-6-R and Shigella sonnei after induction with thiomethyl- $\beta$-D-galactoside. It can also be seen that only a small fraction of this activity is expressed when intact cells are tested. These two strains behave then in a manner analogous to that of the Escherichia coli mutant lac $_{-1}$; i.e. their $\beta$-galactosidase activity is cryptic. Further inspection of table 1 shows that only minute amounts of $\beta$-galactosidase are formed by Shigella flexneri, $S$. dysenteriae strain Sh, and $S$. dysenteriae strain 136-T-5-0. The extremely small amount of $\beta$-galactosidase formed would have escaped detection if incubation with ONPG had not been carried out for prolonged periods of time, in some cases as long as $12 \mathrm{hr}$. Boiled bacteria, incubated with ONPG for the same period of time as the test samples, served as a control for the spontaneous hydrolysis of ONPG. A possible qualitative difference between the $\beta$ galactosidase of $E$. coli and the $\beta$-galactosidase of 
TABLE 1

Occurrence of $\beta$-galactosidase activity in Shigella

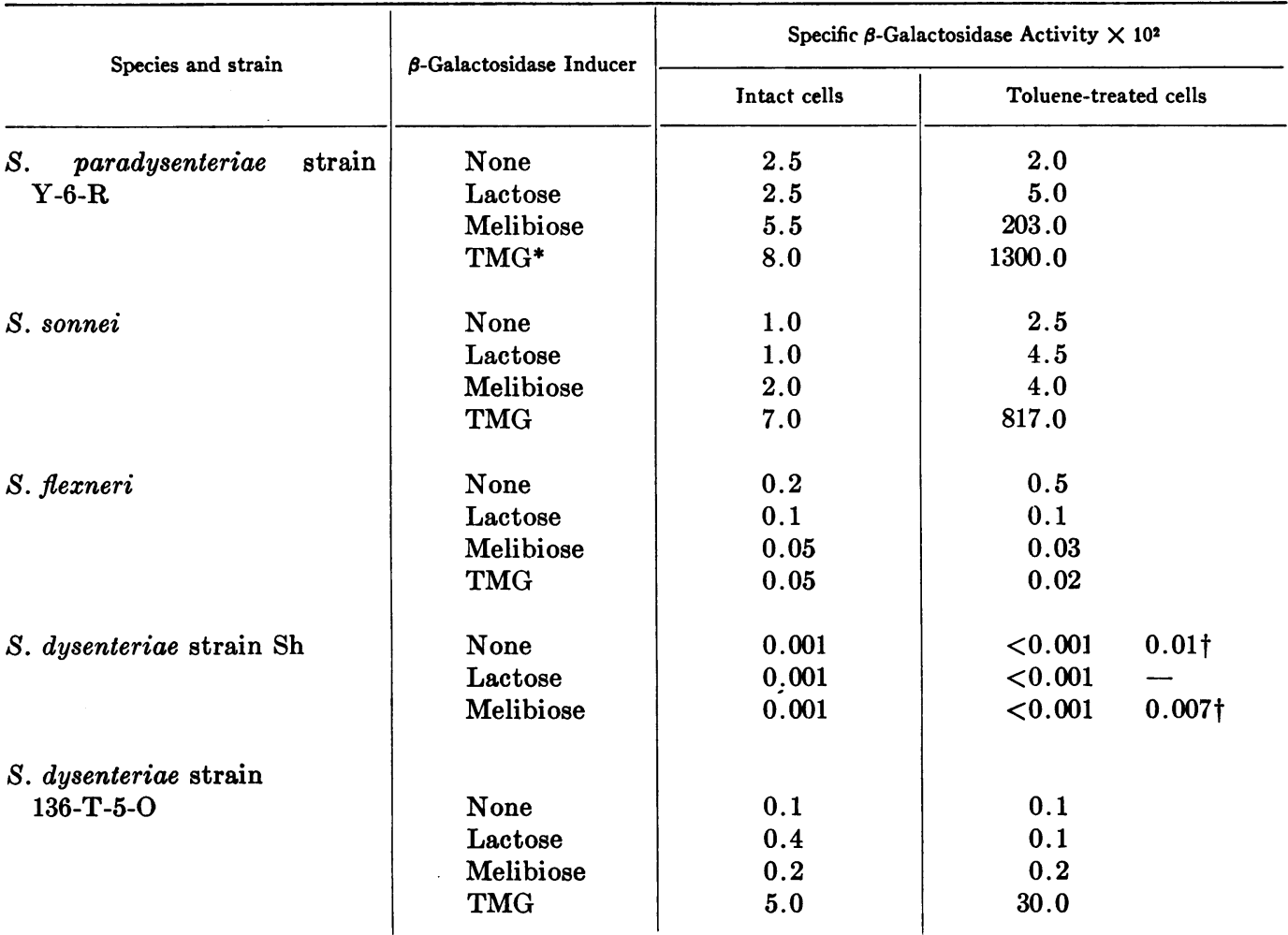

Strain S. paradysenteriae Y-6-R was obtained from Dr. S. A. Zahler of Cornell University. The $S$. sonnei and S. flexneri strains were obtained from Dr. J. Sherris of the University of Washington School of Medicine. Dr. W. L. Barksdale, New York University School of Medicine, furnished the two $S$. dysenteriae strains.

Lactose and melibiose were used at a concentration of 0.5 per cent; thiomethyl- $\beta$-D-galactoside was used at a concentration of $10^{-3} \mathrm{M}$.

Specific $\beta$-galactosidase activity is expressed as micromoles ONPG hydrolized per minute per $\mathrm{mg}$ bacterial protein.

* TMG = thiomethyl- $\beta$-D-galactoside.

$\dagger$ Extracts prepared in sonic oscillator.

S. dysenteriae strains Sh and 136-T-5-0, but not of S. paradysenteriae strain Y-6-R or $S$. sonnei, is suggested by the finding that although $E$. coli, $S$. paradysenteriae, and $S$. sonnei $\beta$-galactosidases were quite resistant to a 60 -min incubation with amyl alcohol, the $S$. dysenteriae strain Sh $\beta$-galactosidase was largely destroyed by treatment with amyl alcohol and the $S$. dysenteriae strain 136T-5-O $\beta$-galactosidase showed intermediate stability. Treatment of strain Sh cells with toluene also destroyed their $\beta$-galactosidase activity. Unpublished work by the author employing strains of Paracolobactrum aerogenoides, Aerobacter aerogenes, and certain $E$. coli mutants suggests that there exist within Enterobacteriaceae strains of bacteria that form $\beta$-galactosidases of widely different stability to a variety of agents.

It may be concluded then that many, if not all, species and strains of the genus Shigella possess the genetic information required for the formation of the enzyme $\beta$-galactosidase. Possibly modification of a pre-existing lac locus by transduction or recombination results in a quantitative and qualitative modification of the ability of the bacteria to form $\beta$-galactosidase. The possibility that the formation of trace amounts of $\beta$-galactosidase in $S$. flexneri and $S$. dysenteriae strain Sh is under the control of a locus other than the classical lac locus has not been excluded, however. 